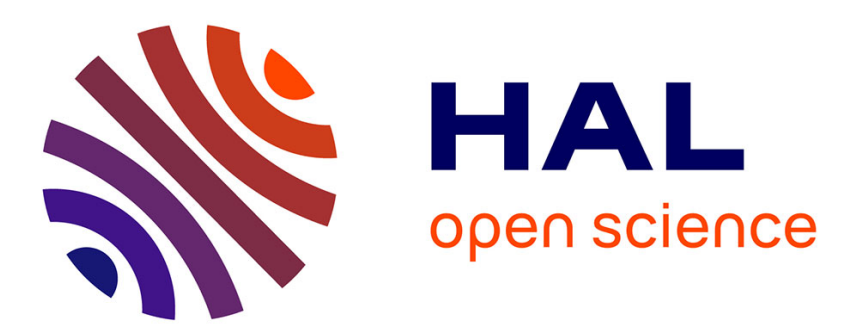

\title{
Proton NMR studies in some cyano-biphenyls, oxy-cyano-biphenyls and PAA at atmospheric pressure \\ Sylvaine Roy
}

\section{To cite this version:}

Sylvaine Roy. Proton NMR studies in some cyano-biphenyls, oxy-cyano-biphenyls and PAA at atmospheric pressure. Journal de Physique II, 1992, 2 (2), pp.219-225. 10.1051/jp2:1992125 . jpa00247625

\section{HAL Id: jpa-00247625 https://hal.science/jpa-00247625}

Submitted on 1 Jan 1992

HAL is a multi-disciplinary open access archive for the deposit and dissemination of scientific research documents, whether they are published or not. The documents may come from teaching and research institutions in France or abroad, or from public or private research centers.
L'archive ouverte pluridisciplinaire HAL, est destinée au dépôt et à la diffusion de documents scientifiques de niveau recherche, publiés ou non, émanant des établissements d'enseignement et de recherche français ou étrangers, des laboratoires publics ou privés. 
Classification

Physics Abstracts

$64.00-61.30$

\title{
Proton NMR studies in some cyano-biphenyls, oxy-cyano- biphenyls and PAA at atmospheric pressure
}

\author{
S. K. Roy \\ Department of Physics, Jadavpur University, Calcutta 700032, India
}

(Received 30 May 1991, accepted 28 October 1991)

\begin{abstract}
Dipolar splitting $\Delta H$ from proton-NMR experiments in 5-8CB, 5-8 OCB and PAA are presented and compared with the quantity $\Sigma$, calculated from refractive index data, in the same set of compounds obtained by Bunning et al. [3]. According to these authors the quantity $\Sigma$ is rather insensitive to the biaxial terms in the ordering matrix and gives the best indication currently available of the temperature dependence of $S_{z z}$ in these materials. $S_{z z}$ can be reliably determined from NMR measurements on suitably deuterated specimens but among the compounds discussed in this paper only 5CB and PAA has so far been studied in this way. The values of $S_{z z}$ for these compounds are compared with $\Sigma$ and the variation is found to be slight (within $3 \%$ ). Because of the involvement of the chain protons, in the proton NMR of the undeuterated specimens it is not obvious that $\Delta H$ should be closely proportional to $S_{z z}$, but for 5CB and PAA it seems to be so (again within $3 \%$ ). For $6 \mathrm{CB}$ too $\Delta H / \Sigma$ is almost constant but for other compounds a systematic rise on cooling is apparent.
\end{abstract}

\section{Introduction.}

The idealized picture of a nematic is of a substance composed of rigid rod-shaped molecules having cylindrical symmetry, the alignment of which can be described by a single order parameter. Real nematics are not so simple ; the molecules are in part flexible and they do not have cylindrical symmetry, so that many order parameters may be needed to describe their mean conformation and alignment completely. In the cyano-biphenyl substances to which this paper principally refers, however there is one order parameter of particular interest. This is the quantity $S_{z z}$, which describes the alignment of an axis which coincides with the C-N bond in the terminal cyano-group, with the $\mathrm{C}-\mathrm{C}$ bond that joins the two phenyl groups which constitute the more or less rigid « core » of the molecule, and with the $\mathrm{C}-\mathrm{C}$ or $\mathrm{C}-\mathrm{O}-\mathrm{C}$ bonds that link this core to a flexible alkyl tail.

$S_{z z}$ can be reliably determined from NMR measurements on suitably deuterated specimens but of the cyano-biphenyls only $5 \mathrm{CB}$ has so far been studied in this way [1]. Many authors interested in $S_{z z}$ have therefore estimated it from the bulk properties such as birefringence, the magnetic anisotropy, or the anisotropy in relative permeability. An assumption is made that the chosen property is proportional to $S_{z z}$, and the constant of proportionality is 
estimated by a procedure first used by Haller [2]. Bunning et al. [3] who have examined in some detail the effect of biaxial terms in the ordering matrix, conclude that the birefringence represented by a quantity which they call $\Sigma\left(\Sigma=\left(n_{\mathrm{e}}^{2}-n_{\mathrm{o}}^{2}\right) /\left(\overline{n^{2}}-1\right), n_{\mathrm{e}}\right.$ and $n_{\mathrm{o}}$ are the extraordinary and ordinary refractive indices and $\left.\overline{n^{2}}=\left(n_{\mathrm{e}}^{2}+2 n_{0}^{2}\right) / 3\right)$ is less sensitive to the terms than $\Delta \chi$, and $\Sigma$ is therefore the better indicator of $S_{z z}$. They plot a graph which suggests that for $5 \mathrm{CB}$ the ratio of $\Sigma$ to $S_{z z}$ varies by less than $3 \%$ between $T_{\mathrm{NI}}$ and the lowest temperature at which measurements on the nematic phase are still feasible. They tabulate experimental results for the ordinary and extraordinary refractive indices of four alkyl-cyanobiphenyls other than 5CB and for four alkoxy-cyano-biphenyls and suggests that the values of $\Sigma$ deduced from the results give the best indication currently available of the temperature dependence of $S_{z z}$ in all these materials. Incidentally, they also tabulate refractivity results for p-azoxy-anisole (PAA). This is another material for which « chain-deuterated s specimens have been studied by NMR [4,5] and had Bunning et al. plotted the ratio of $\Sigma$ to $S_{22}$ for PAA they would have found that its variation with temperature is as slight as it is in $5 \mathrm{CB}$.

The object of this paper is to present results covering the same range of materials for the proton NMR linewidth measured using undeuterated specimens. A derivative plot for a typical proton resonance line is shown in figure 1 , and the linewidth or the mean dipolar splitting $\Delta H$ is marked on the figure as the distance between zeros which correspond to the two principal maxima of the line. The splitting is partly due to interactions between the protons attached to the bi-phenyl core but interactions involving protons attached to carbon atoms in the alkyl tail, especially those carbon atoms which are adjacent to the core, are thought to contribute significantly towards it (see section 4 below). Because chain protons are involved, it is not obvious that $\Delta H$ should be closely proportional to $S_{z z}$, but for the both $5 \mathrm{CB}$ and PAA it seems to be so, at any rate within $3 \%$. Thus the data to be presented provide for other cyano-biphenyls, an indication of the temperature dependence of $S_{z z}$ that may be as accurate as the indication provided by birefringence measurements. It is obviously of interest to compare the two.

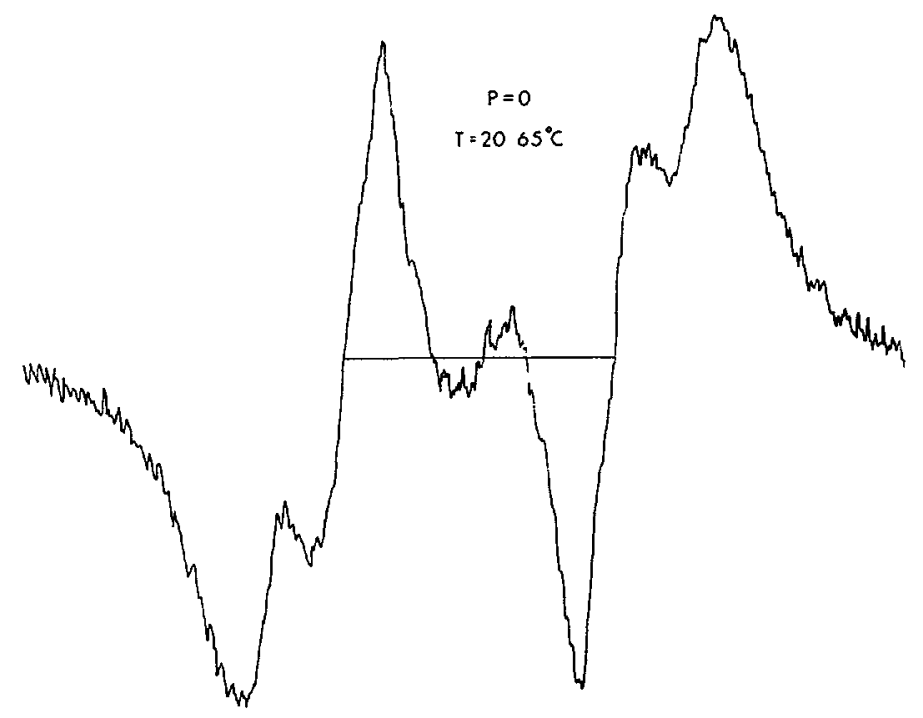

Fig. 1. Derivative line shape in $7 \mathrm{CB}$ at $T=20.65^{\circ} \mathrm{C}$. 


\section{Experimental method.}

The proton resonance lines were recorded at atmospheric pressure, using the same spectrometer as was used for measurements at high pressures which have been described in a previous paper [6]. It operated at a frequency of $27.1 \mathrm{MHz}$. The specimen was contained in a glass cell which also contained the if coil and after degassing this cell was sealed. It was surrounded by an oil bath, the temperature of which can be controlled and measured to within $\pm 0.05 \mathrm{~K}$.

\section{Results.}

The line shape varied in some particulars from one material to the next, the most significant feature of this variation being an odd-even oscillation in the size of the central peak; this peak was much bigger for $6 \mathrm{CB}$ and $8 \mathrm{CB}$ than it was for $5 \mathrm{CB}$ and $7 \mathrm{CB}$ and it was much bigger for $50 \mathrm{CB}$ and $7 \mathrm{OCB}$ than it was for $60 \mathrm{CB}$ and $80 \mathrm{CB}$. An explanation for the oscillation has been given by Weber [7] and it does not merit further discussion here. Wallis and Roy [6] have noted that for a single substance the line shape is liable to vary with pressure at constant temperature and at constant (atmospheric) pressure it is also liable to vary with temperature ; further details are available in Roy [8]. For PAA the line shape was in good agreement with that observed for non-deuterated specimens by Visintainer et al. [9] and Limmer et al. [4].

Wallis and Roy [6] fitted thier high pressure results for $\Delta H$ in $6 \mathrm{CB}$ and $7 \mathrm{CB}$ by polynomials in $p$ and $T$, but these polynomials are not to be trusted when $p$ is effectively zero and the atmospheric results are presented graphically here, in figures $2 a$ and $2 b$. The high degree of reproducibility is indicated by the small scatter of the points in these figures.

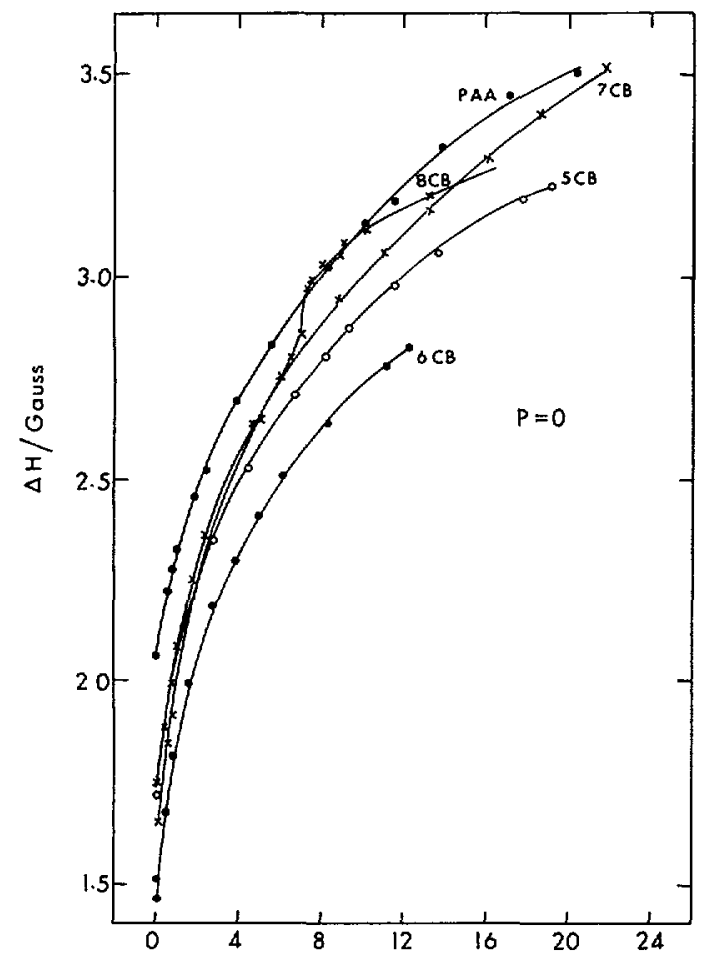

a)

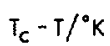

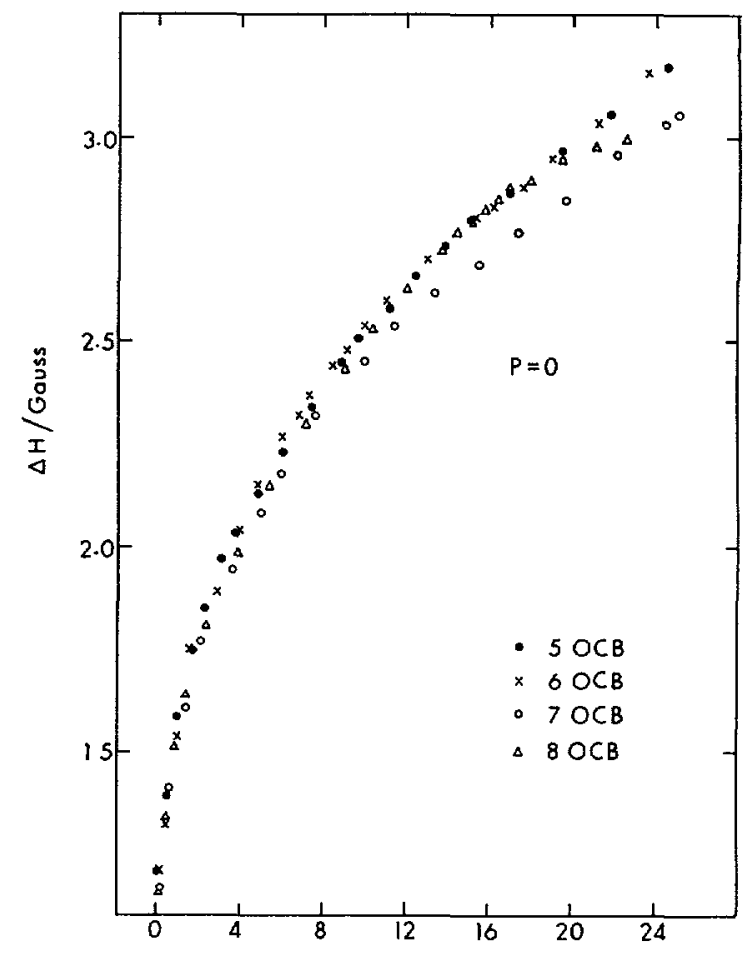

b)

$$
\mathrm{T}_{\mathrm{C}}-\mathrm{T} /{ }^{\circ} \mathrm{K}
$$

Fig. 2. - Dipolar splitting $\Delta H$ plotted against $\left(T_{\mathrm{N} 1}-T\right)$ in 5-8CB, 5-8OCB and PAA. 


\section{Discussion.}

We must first substantiate the claim made in the introduction, that the ratio of $\Delta H$ to $S_{2 z}$ is essentially independent of temperature for both 5CB and PAA. Table I lists the values of $S_{z z}$ given for $5 \mathrm{CB}$ by Emsley et al. [1], together with corresponding values for $\Sigma$ deduced from the table in Bunning $e t$ al. [3] and corresponding values for $\Delta H$ read off from the smooth curve that is drawn through the point for $5 \mathrm{CB}$ in figure $2 \mathrm{a}$. Values for both $\Sigma / S_{z z}$ and $S_{z z} / \Delta H$ are given in the final columns. Table II contains similar results relating to PAA; the values for $S_{z z}$ in this table come from the measurements on chain-deuterated specimens reported by Rowell et al. [5] and Limmer et al. [4]. The temperature dependence of both ratios is evidently slight for both substances. In the case of 5CB both ratios seem anomalously low at $\left(T_{\mathrm{NI}}-T\right)=1.5 \mathrm{~K}$ and anomalously high at $\left(T_{\mathrm{NI}}-T\right)=6 \mathrm{~K}$, but since

Table I. - Comparison of $\Delta H$ from $\mathrm{H}^{1}-\mathrm{NMR}$ of $5 \mathrm{CB}$ with $S_{z z}$ from $5 \mathrm{CB}-\mathrm{d}_{15}$ reported by Emsley et al. [1] and $\Sigma$ from Bunning et al. [3].

\begin{tabular}{|c|c|c|c|c|c|}
\hline$\left(T_{\mathrm{NI}}-T\right) / \mathrm{K}$ & $S_{z z}$ & $\Sigma$ & $\Delta H / \mathrm{Gauss}$ & $\Sigma / S_{z z}$ & $S_{z z} / \Delta H /$ Gauss \\
\hline 0 & 0.338 & 0.218 & 1.72 & 0.645 & 0.197 \\
0.5 & 0.384 & 0.246 & 1.90 & 0.641 & 0.202 \\
1.0 & 0.414 & 0.265 & 2.08 & 0.640 & 0.199 \\
1.5 & 0.443 & 0.280 & 2.18 & 0.632 & 0.203 \\
3.5 & 0.491 & 0.317 & 2.44 & 0.646 & 0.201 \\
6.0 & 0.524 & 0.346 & 2.66 & 0.660 & 0.197 \\
8.5 & 0.565 & 0.366 & 2.82 & 0.648 & 0.200 \\
10.5 & 0.583 & 0.379 & 2.93 & 0.650 & 0.199 \\
13.0 & 0.599 & 0.393 & 3.05 & 0.656 & 0.196 \\
15.5 & 0.615 & 0.405 & 3.13 & 0.659 & 0.196 \\
\hline
\end{tabular}

Table II. - Comparison of $\Delta H$ from $\mathrm{H}^{1}-\mathrm{NMR}$ of PAA with $S_{z 2}$ from $\mathrm{PAA}-\mathrm{d}_{6}$ reported by Limmer et al. [4] and Rowell et al. [5] and $\Sigma$ from Bunning et al. [3].

\begin{tabular}{|c|c|c|c|c|c|}
\hline$\left(T_{\mathrm{NI}}-T\right) / \mathrm{K}$ & $S_{z z}$ & $\Sigma$ & $\Delta H /$ Gauss & $\Sigma / S_{z z}$ & $S_{z z} / \Delta H /$ Gauss \\
\hline 1.5 & 0.393 & 0.371 & 2.38 & 0.944 & $0.165[5]$ \\
2.0 & 0.391 & 0.382 & 2.46 & 0.977 & 0.159 \\
3.0 & 0.400 & 0.402 & 2.51 & 1.005 & 0.159 \\
4.0 & 0.415 & 0.417 & 2.71 & 1.005 & 0.153 \\
5.0 & 0.437 & 0.431 & 2.79 & 0.986 & 0.156 \\
7.0 & 0.496 & & 2.95 & & $0.168[5]$ \\
10.0 & 0.506 & 0.479 & 3.12 & 0.947 & 0.162 \\
12.0 & 0.525 & 0.494 & 3.22 & 0.941 & 0.163 \\
12.25 & 0.539 & & 3.24 & & $0.167[5]$ \\
14.0 & 0.538 & 0.507 & 3.32 & 0.942 & 0.162 \\
16.0 & 0.554 & 0.519 & 3.39 & 0.937 & 0.163 \\
17.0 & 0.568 & & 3.43 & & $0.166[5]$ \\
\hline
\end{tabular}


the same anomalies show up in the relation $\Delta \chi / S_{z z}$ (see Bunning et al.), it is probable that unsuspected errors in the results for $S_{z z}$ at these two temperatures are to blame.

For PAA the ratio $\Delta H / S_{z z}$ is evidently very close to the value of 6.17 Gauss used by Mckee and McColl [10] who based their calculation on Weber's [7] work. According to Visintainer $e t$ al. [9] this ratio is about $20 \%$ smaller for «chain-deuterated »PAA (the « chains » in PAA being the methoxy groups at the two ends of the molecule). The difference indicates the importance of interactions involving the methoxy protons in this material.

For $5 \mathrm{CB}$ the ratio is clearly about 5 Gauss. To judge from the result quoted by Emsley $e t$ al. [11] for the proton line-width in chain-deuterated 5CB (this result corresponds to about 1 Gauss at a temperature of $31^{\circ} \mathrm{C}$ ), the ratio would be only about 2 Gauss in the absence of interactions involving protons on the pentyl chain. There are of course 11 of these protons as opposed to 6 protons in the methoxy groups in a PAA molecule, but the fact that chaindeuteration has a greater effect on $\Delta H$ in 5CB than it does in PAA is no doubt due to the oxygen atoms in the latter material, which keep the chain and the ring protons apart. Comparison of the figures $2 \mathrm{a}$ and $2 \mathrm{~b}$ show that, for a given value of $\left(T_{\mathrm{NI}}-T\right)$, $\Delta H$ tends to be less for an alkoxy-cyanobiphenyl than for the corresponding alkylcyanobiphenyl by about 0.5 Gauss and the oxygen atoms are no doubt responsible for this difference too.

We now turn to the correlation between $\Delta H$ and $\Sigma$, not just for PAA and 5CB but for all other members of the two cyanobiphenyl families. It is illustrated by the curves for $\Delta H / \Sigma$ plotted in figures $3 a$ and $3 b$.

All the curves in these figures bend upwards quite sharply as $T_{\mathrm{NI}}$ is approached, an effect which was noted by Wallis and Roy [6] in their discussion of their high pressure results for 5CB. A satisfactory explanation for this phenomenon is still not available. Several authors including Limmer et al. [4] have pointed out that disorder due to the director fluctuations which persists for times longer than $\Delta \omega^{-1}$, where $\Delta \omega$ is the frequency range that corresponds to $\Delta H$, has a different effect upon NMR line shapes than disorder which persists for shorter times and according to Limmer et al. [4] the difference is more noticeable within a few degrees of $T_{\mathrm{NI}}$ than at lower temperatures. Perhaps this has something to do with the bending near $T_{\mathrm{NI}}$.

For $T_{\mathrm{NI}}-T>$ about $2 \mathrm{~K}$, the curves are remarkably level for both $5 \mathrm{CB}$ and PAA, which is of course no surprise, in view of the figures in tables I and II. The curve for $6 \mathrm{CB}$ is almost equally level. For all other materials, however, a systematic rise on cooling is apparent, and in the most marked case (8OCB) $\Delta H / \Sigma$ rises by as much as $10 \%$. Opinions will no doubt differ as to whether the proportionality is more exact for $\Delta H$ than for $\Sigma$, or vice versa.

Before we end this discussion, it must be pointed out that any small difference in $T_{\mathrm{NI}}$ obtained in the two experiments, namely the proton NMR and the optical birefringence could possibly lead to an anomalous behaviour of $\Delta H / \Sigma$. We would however, like to make it clear that the two experiments were carried out in the same laboratory using samples obtained from the $\mathrm{BDH}$ and that the transition temperatures recorded for the two experiments were always within the limits of accuracy of the temperature measurements, i.e., $\mp 0.05 \mathrm{~K}$. The identical behaviour of $\Delta H / \Sigma$ close to $T_{\mathrm{NI}}$ for all samples, could only arise from a systematic difference in the values of $T_{\mathrm{NI}}$ for all samples in the two experiments. No such systematic difference in the $T_{\mathrm{NI}}$ 's were observed and hence it can safely be concluded that the behaviour of $\Delta H / \Sigma$ close to the clearing point is by no means an artifact of the renormalization of the temperature scales in the two experiments.

Finally, we would like to make a comment on the existence of a small biphasic region near $T_{\mathrm{NI}}$ in the two experiments and its possible consequence on $\Delta H / \Sigma$. The experiments were performed with adequate care so that the coexistence of the $\mathrm{N}-\mathrm{I}$ phase could easily be 


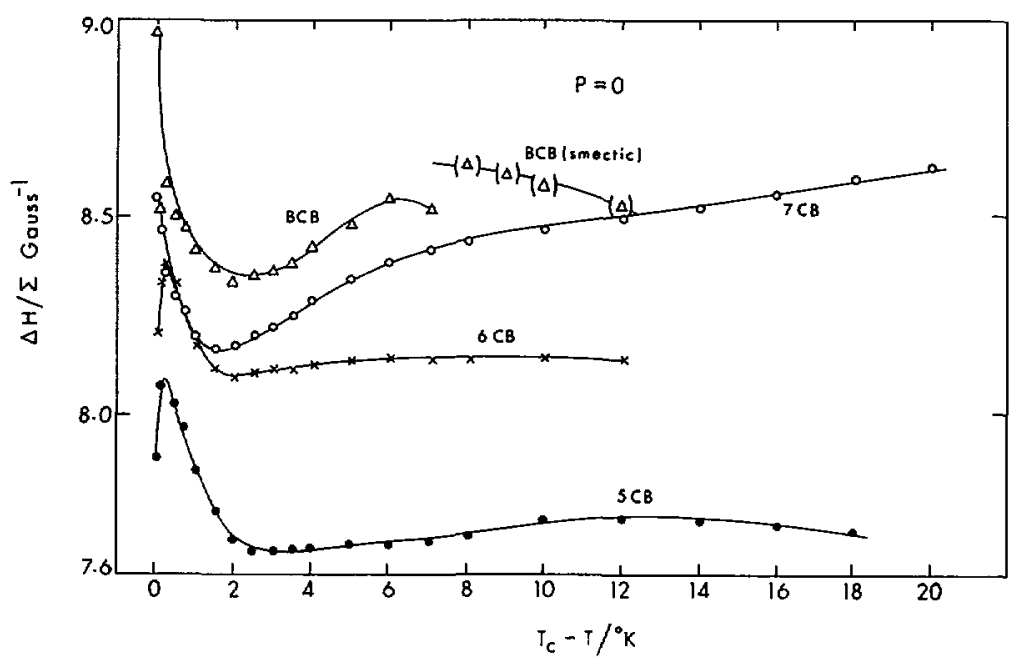

a)

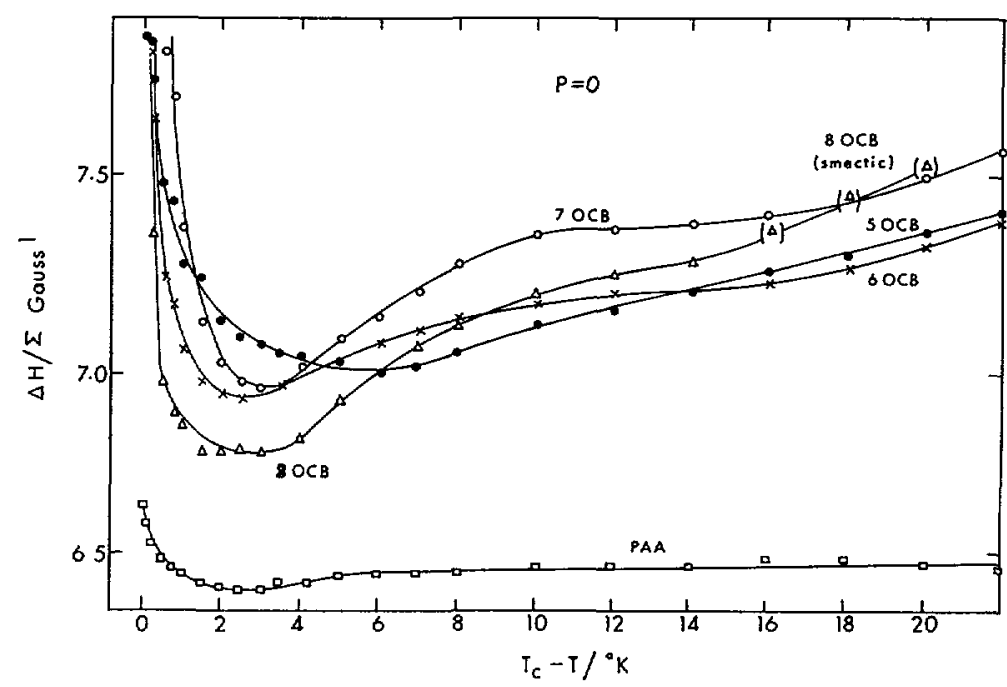

b)

Fig. 3. $-\Delta H / \Sigma$ plotted against $\left(T_{\mathrm{NI}}-T\right)$ in $5-8 \mathrm{CB}, 5-8 \mathrm{OCB}$ and PAA.

detected in the NMR experiment, the appearance of a sharp central peak indicated the formation of the isotropic phase and, as a matter of fact, this helped us to record $T_{\mathrm{NI}}$ with great accuracy. Clearly this would not affect $\Delta H$ in any way. In the birefringence experiment too, the splitting between the two spots of the laser beam (Chatelain Wedge method [12]) - which leads to the determination of $\Sigma$ - was unaffected by the appearance of a central spot, which again, was an accurate indication of the N-I transition point and certainly had no effect on the value of $\Sigma$ at this temperature.

\section{Acknowledgment.}

The author is grateful to Dr. T. E. Faber and Dr. D. Roy for their valuable comments during the preparation of the paper. 


\section{References}

[1] Emsley J. W., Luckhurst G. R. and Stockley C. P., Mol. Phys. 44 (1981) 565.

[2] Haller I., Progr. Solid State Chem. 10 (1975) 103.

[3] Bunning J. D., Crellin D. A. and Faber T. E., Liq. Crys. 1 (1986) 37.

[4] Limmer St., Schmiedel H., Hillner B., Losche A. and Grande S., J. Phys. France 41 (1980) 869.

[5] Rowell J. C., Phillips W. D., Melby L. R, and Panar M., J. Chem. Phys, 43 (1965) 3442.

[6] Wallis G. P. and RoY S. K., J. Phys. France 41 (1980) 1165.

[7] Weber K. H., Ann. Phys. 3 (1959) 1.

[8] RoY S. K., Ph. D. Thesis, Cambridge University (1981).

[9] Visintainer J. J., Bock E., Dong R. Y. and Tomchuk E., Can. J. Phys. 53 (1975) 1483.

[10] MCkee T. J. and MCColl J. R., Phys. Rev. Lett. 34 (1975) 1076.

[11] Emsley J. W., Lindon J. C. and Luckhurst G. R., Mol. Phys. 30 (1975) 1913.

[12] Pellete O. and Chatelain P., Bull. Soc. Franc. Mineral. 73 (1950) 154. 